\title{
Data Element Identifier
}

National Cancer Institute

\section{Source}

National Cancer Institute. Data Element Identifier. NCI Thesaurus. Code C142474.

Information associated with a data element that includes the origin of the data element, the date and time of entry, and the identification number of the study subject to whom the data element applies. (FDA) 